(2) Open Access Full Text Article

\title{
Assessing the potential value of long interspersed element-I hypomethylation in colorectal cancer: evidence from retrospective studies
}

This article was published in the following Dove Press journal:

OncoTargets and Therapy

5 November 2015

Number of times this article has been viewed

\author{
Jie-Ting Tang ${ }^{1,2}$ \\ Zhen-Hua Wang ${ }^{1,2}$ \\ Jing-Yuan Fang ${ }^{1,2}$
}

'Division of Gastroenterology and Hepatology, Renji Hospital, ${ }^{2}$ Shanghai Institute of Digestive Disease, School of Medicine, Shanghai Jiao

Tong University, Shanghai, People's Republic of China
Correspondence: Jing-Yuan Fang Division of Gastroenterology and Hepatology, Renji Hospital, Shanghai Institute of Digestive Disease, School of Medicine, Shanghai Jiao Tong University, I 45 Middle Shandong Road, Shanghai 20000I, People's Republic of China Tel +86 2I 53882450

$\mathrm{Fax}+862163266027$

Email jingyuanfang@sjtu.edu.cn
Background and aims: Long interspersed element-1 (LINE-1) hypomethylation may play an important role in colorectal cancer (CRC). Studies were identified that investigated LINE-1 methylation levels in CRC compared with normal controls.

Methods: The random-effects model was used to estimate standardized mean difference with $95 \%$ confidence intervals according to the heterogeneity between the studies. We explored the relationship between LINE-1 hypomethylation and microsatellite instability (MSI) status, clinical features, and molecular features in CRC patients using a fixed-effects model.

Results: A total of 7396 CRC patients were included in the meta-analysis. LINE-1 methylation was significantly lower in CRC patients than in controls $(P=0.000)$. Mean LINE-1 methylation was significantly lower in non-MSI-high than in MSI-high tumors $(P=0.000)$. LINE-1 hypomethylation was found more frequently in patients with a family history compared with those without family history $(P=0.002)$. Patients with left colon cancer had lower LINE-1 methylation than those with right colon cancer $(P=0.001)$. LINE-1 methylation was not associated with body mass index or patient sex. LINE-1 hypomethylation was found in p21 lost tumors $(P=0.000)$. LINE-1 methylation levels were not associated with KRAS or PIK3CA-mutation status.

Conclusion: LINE-1 hypomethylation is a potential biomarker for risk of CRC and associated with various clinical and molecular features of CRC.

Keywords: LINE-1, hypomethylation, colorectal cancer, MSI

\section{Introduction}

Epigenomic alterations, including DNA methylation, histone acetylation, and RNA editing are important mechanisms during human carcinogenesis. ${ }^{1-3}$ A considerable number of DNA methylation occurs in repetitive sequences. Long interspersed element-1 (LINE-1) is an interspersed repetitive DNA retrotransposon leading to genomic instability in cancer cells via influencing gene expression status. ${ }^{4}$ It duplicates via a copy-and-paste mechanism and rearranges the genome continuously. Its promoter is internally located in $5^{\prime}$ untranslated region ranging from +1 to $909 \mathrm{bp} .^{5}$ The methylation status of LINE-1 was first demonstrated in cancer cell lines in 1993. ${ }^{6}$ LINE-1 hypomethylation was detected in head and neck squamous cell carcinoma, bladder cancer, gastric cancer, and so on, and was demonstrated to be associated with poor prognosis. ${ }^{7-10}$ Epigenetic changes of LINE-1 occur early during the process of carcinogenesis. LINE-1 methylation levels were found to be nearly normally distributed in CRC patients. Studies showed that LINE-1 methylation levels seemed to be checkered in CRC cell lines. ${ }^{11,12}$ Recently, an increasing number of studies have evaluated LINE-1 methylation levels in association with tumor progression of CRC. However, some of 
the results remain controversial in several studies. ${ }^{11,13}$ In a previous meta-analysis, LINE-1 hypomethylation in blood and tissues was evaluated in several types of cancer including CRC. ${ }^{14}$ Nevertheless, the results remain insufficient, and the role of LINE-1 hypomethylation in specific subgroups is needed to be better clarified. Hence, we conducted a metaanalysis to pool available trial data to more precisely estimate the values of LINE-1 hypomethylation in CRC patients.

\section{Materials and methods}

\section{Search strategy}

Systematic computerized searches of publications before January 2015 were performed using the PubMed, Embase, and the Web of Science databases. For both of the databases, keywords of ("colorectal cancer [CRC]" or "colorectal carcinoma") AND ("LINE-1" or "long interspersed element-1") AND ("hypomethylation" or "methylation") were used to search eligible studies. Moreover, the bibliographies from selected articles and review articles were scrutinized to trace other potentially relevant studies.

\section{Inclusion criteria and data extraction}

Studies were included in the quantitative analysis only if they satisfied the following criteria: (1) case-control design, and (2) studies with available mean values and standard deviations (SDs) of LINE-1 methylation levels. The first two authors independently reviewed the papers for eligibility and quality. Disagreements were resolved by the third author. Data, including the first author's name, year of publication, ethnicity, total number of cases and controls, testing methods for methylation analysis, testing materials in each study, and mean values and the SD of global DNA methylation levels for each group were collected.

\section{Statistical analysis}

The Stata version 11.0 software (StataCorp LP, College Station, TX, USA) was used for all data analyses. Heterogeneity of effect sizes across the studies was examined using the $Q$ statistic. If the $P$-value was $<0.05$, then the random-effects model (DerSimonian and Laird method) was used to estimate the standardized mean difference (SMD) with 95\% confidence interval (CI). ${ }^{15}$ Otherwise, a fixed-effects model (Mantel-Haenszel method) was used. Additionally, the magnitude of the between-study heterogeneity was also assessed by the $I^{2}$ value. An $I^{2}$ value of $0 \%$ denoted no observed heterogeneity, whereas $25 \%$ was "low", $50 \%$ was "moderate", and 75\% was "high" heterogeneity. ${ }^{16}$ Furthermore, subgroup analyses were conducted based on ethnicity, and by methods used to measure global DNA methylation levels.
Sensitivity analyses were performed to assess the contributions of single studies to the final results.

Funnel plots with the Begg's test ${ }^{17}$ were visually evaluated for any asymmetry to detect a possible publication bias, in which a $P$-value $<0.10$ was considered representative of a statistically significant bias. ${ }^{18}$ Trim and fill analysis was applied if there was publication bias. ${ }^{19}$

\section{Results}

\section{Characteristics of the included studies}

Fourteen studies ${ }^{10,12,20-31}$ were identified according to our search strategy and study selection criteria in the final metaanalysis. In five studies, ${ }^{12,20-22,24}$ LINE-1 methylation levels were evaluated both in tumor and in normal controls tissues, among which, two papers ${ }^{20,24}$ included different cancer types, which were separated into substudies. The remaining ten studies $^{10,23,25-32}$ included only CRC patients. The characteristics of all included studies are summarized in Table 1.

\section{LINE-I methylation levels decreased in CRC}

LINE-1 methylation levels were successfully measured both in cancer and in normal controls tissues in five studies, ${ }^{12,20-22,24}$ including a total of 693 CRC samples. LINE-1 methylation levels were significantly lower in cancer patients than in the control samples (SMD: -2.26 ; 95\% CI: $-3.08,-1.45 ; z=5.44$, $P=0.000$, Figure 1).

However, heterogeneity between studies was significantly high $\left(I^{2}=96.6 \%\right)$; thus, subgroup analysis based on ethnicity was performed. A total of ten studies ${ }^{12,20,22,24}$ (including substudies) were from non-Asian, and one ${ }^{21}$ from Asian countries. Methylation levels of LINE-1 were significantly lower in CRC patients than in control samples in the non-Asian subgroup (SMD: -2.48 ; $95 \%$ CI: $-3.28,-1.68 ; P=0.000)$. Heterogeneity between studies, in the non-Asian subgroup, was still significantly high $\left(I^{2}=95.9 \%\right)$ (Figure 2$)$.

Subgroup analysis based on methods used to measure global DNA methylation levels was performed. Pyrosequencing was used in a total of six studies (including substudies), whereas combined bisulfite restriction analysis (COBRA) was used in four studies. Only one study $^{24}$ used multicolor MethyLight assay to evaluate LINE-1 methylation level. The SMDs for pyrosequencing and COBRA LINE-1 subgroups were -3.53 (95\% CI: $-4.76,-2.30 ; P=0.000)$ and $-1.11(95 \% \mathrm{CI}:-1.60,-0.62$; $P=0.005)$, respectively. Heterogeneity between studies was also significantly high ( $I^{2}=96.6 \%$ and $76.3 \%$, respectively) (Figure 3). 
Table I Main characteristics of studies included in the meta-analysis

\begin{tabular}{|c|c|c|c|c|c|c|}
\hline Study & Year & Ethnicity & Tumor cases & Normal controls & Methods & Materials \\
\hline Antelo et al (early onset) ${ }^{20}$ & 2012 & Non-Asian & 185 & 32 & Pyrosequencing & FFPE \\
\hline Antelo et al (Lynch syndrome) ${ }^{20}$ & 2012 & Non-Asian & 20 & 32 & Pyrosequencing & FFPE \\
\hline Antelo et al (older onset sporadic MSI) ${ }^{20}$ & 2012 & Non-Asian & 46 & 32 & Pyrosequencing & FFPE \\
\hline Antelo et al (older onset sporadic MSS) ${ }^{20}$ & 2012 & Non-Asian & 89 & 32 & Pyrosequencing & FFPE \\
\hline Matsunoki et al ${ }^{21}$ & 2012 & Asian & 48 & 48 & Multicolor MethyLight assay & FFPE \\
\hline Hur et $\mathrm{al}^{22}$ & 2014 & Non-Asian & 77 & 77 & Pyrosequencing & FFPE \\
\hline Rhee et $\mathrm{al}^{23}$ & 2012 & Asian & 207 & NA & Pyrosequencing & FFPE \\
\hline Estecio et $\mathrm{al}^{12}$ & 2007 & Non-Asian & 60 & 60 & Pyrosequencing & Fresh tissues \\
\hline Pavicic et al (sporadic MSS) ${ }^{24}$ & 2012 & Non-Asian & 55 & 55 & COBRA & FFPE \\
\hline Pavicic et al (sporadic MSI) ${ }^{24}$ & 2012 & Non-Asian & 52 & 52 & COBRA & FFPE \\
\hline Pavicic et al (Lynch syndrome) ${ }^{24}$ & 2012 & Non-Asian & 43 & 43 & COBRA & FFPE \\
\hline Pavicic et al $(\mathrm{FCCX})^{24}$ & 2012 & Non-Asian & 18 & 18 & COBRA & FFPE \\
\hline Imamura et $\mathrm{al}^{25}$ & 2014 & Non-Asian & 1,267 & NA & Pyrosequencing & FFPE \\
\hline Morikawa et $\mathrm{a}^{26}$ & 2012 & Non-Asian & 1,060 & NA & Pyrosequencing & FFPE \\
\hline Shima et $\mathrm{al}^{27}$ & 2011 & Non-Asian & 1,072 & NA & Pyrosequencing & FFPE \\
\hline Baba et $\mathrm{al}^{10}$ & 2010 & Non-Asian & 869 & NA & Pyrosequencing & FFPE \\
\hline Hazra et $\mathrm{a}^{28}$ & 2010 & Non-Asian & 182 & NA & Pyrosequencing & FFPE \\
\hline Ogino et $\mathrm{a}^{29}$ & 2009 & Non-Asian & 647 & NA & Pyrosequencing & FFPE \\
\hline Ogino et $\mathrm{a}^{30}$ & 2009 & Non-Asian & 82 & NA & Pyrosequencing & FFPE \\
\hline Lou et $\mathrm{a}^{31}$ & 2015 & Asian & 129 & NA & Pyrosequencing & FFPE \\
\hline Yamauchi et al ${ }^{32}$ & 2012 & Non-Asian & 1188 & NA & Pyrosequencing & FFPE \\
\hline
\end{tabular}

Abbreviations: COBRA, combined bisulfite restriction analysis; FCCX, familial colorectal cancer type X; FFPE, formalin fixed paraffin embedded; MSI, microsatellite instability; MSS, microsatellite stability; NA, not available.

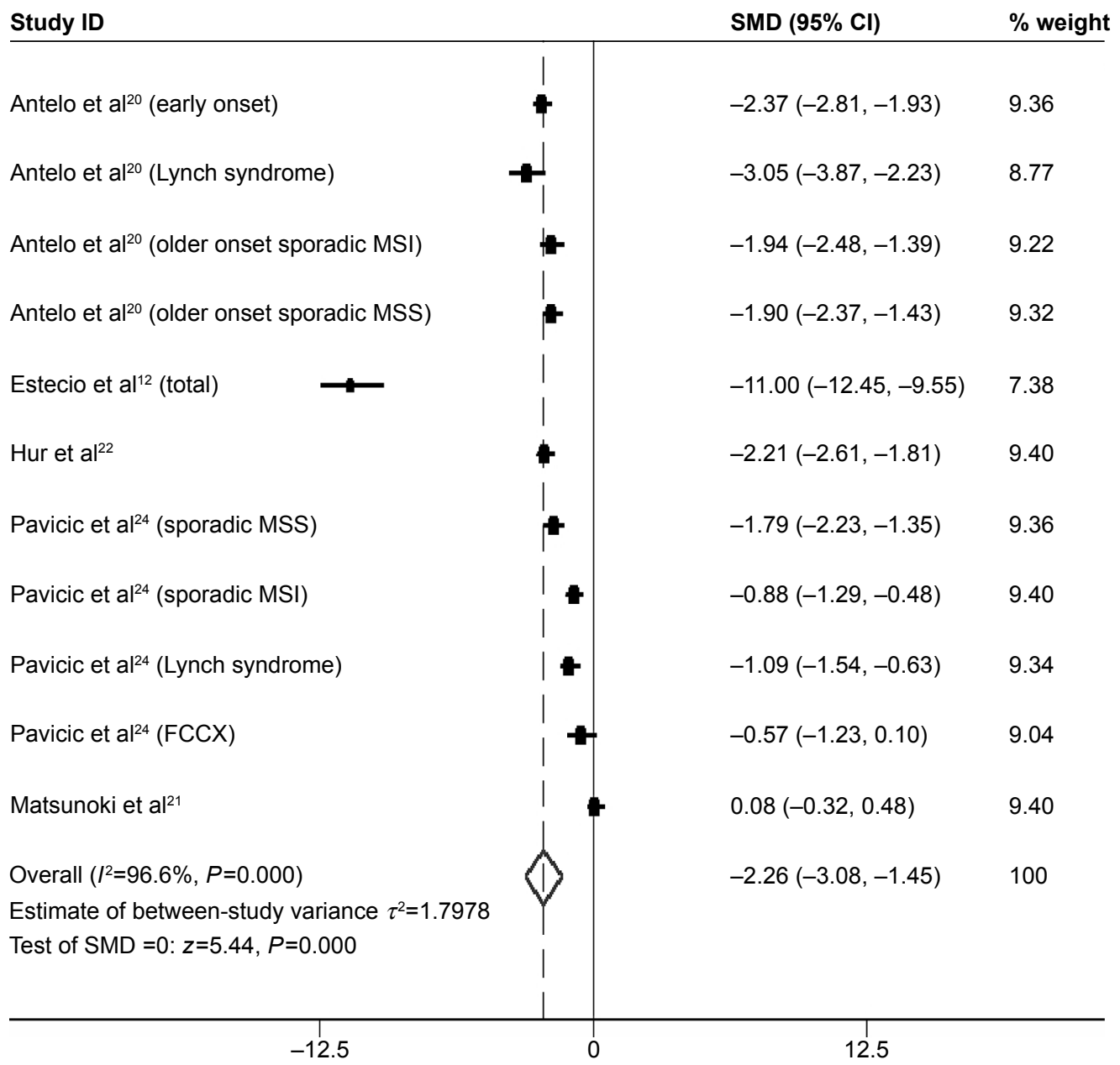

Figure I Forest plot of the SMD of LINE-I methylation levels between colorectal cancer and control groups.

Note: Weights are from random effects analysis.

Abbreviations: $\mathrm{Cl}$, confidence interval; FCCX, familial colorectal cancer type X; LINE-I, long interspersed element-I; MSI, microsatellite instability; MSS, microsatellite stability; SMD, standardized mean difference. 


\begin{tabular}{|c|c|c|}
\hline Study ID & SMD (95\% Cl) & $\%$ weight \\
\hline \multicolumn{3}{|l|}{ Non-Asian } \\
\hline Antelo et al ${ }^{20}$ (early onset) & $-2.37(-2.81,-1.93)$ & 9.36 \\
\hline Antelo et $\mathrm{al}^{20}$ (Lynch syndrome) & $-3.05(-3.87,-2.23)$ & 8.77 \\
\hline Antelo et $\mathrm{al}^{20}$ (older onset sporadic MSI) & $-1.94(-2.48,-1.39)$ & 9.22 \\
\hline Antelo et $\mathrm{a}^{20}$ (older onset sporadic MSS) & $-1.90(-2.37,-1.43)$ & 9.32 \\
\hline Estecio et al ${ }^{12}$ (total) & $-11.00(-12.45,-9.55)$ & 7.38 \\
\hline Hur et $\mathrm{al}^{22}$ & $-2.21(-2.61,-1.81)$ & 9.40 \\
\hline Pavicic et al ${ }^{24}$ (sporadic MSS) & $-1.79(-2.23,-1.35)$ & 9.36 \\
\hline Pavicic et al24 (sporadic MSI) & $-0.88(-1.29,-0.48)$ & 9.40 \\
\hline Pavicic et al ${ }^{24}$ (Lynch syndrome) & $-1.09(-1.54,-0.63)$ & 9.34 \\
\hline Pavicic et $\mathrm{al}^{24}$ (FCCX) & $-0.57(-1.23,0.10)$ & 9.04 \\
\hline Subtotal $\left(I^{2}=95.9 \%, P=0.000\right)$ & $-2.48(-3.28,-1.68)$ & 90.60 \\
\hline \multicolumn{3}{|l|}{ Asian } \\
\hline Matsunoki et $\mathrm{al}^{21}$ & $0.08(-0.32,0.48)$ & 9.40 \\
\hline Subtotal $\left(I^{2}=. \%, P=.\right)^{*}$ & $0.08(-0.32,0.48)$ & 9.40 \\
\hline Overall $\left(I^{2}=96.6 \%, P=0.000\right)$ & $-2.26(-3.08,-1.45)$ & 100 \\
\hline
\end{tabular}

Figure 2 Forest plot of the SMD of LINE-I methylation levels between colorectal cancer and control groups.

Note: The subgroup analysis was based on ethnicity. Weights are from random effects analysis. *There is only one study in the Asian subgroup, so the $I^{2}$ of the subgroup is blank in the output graph.

Abbreviations: FCCX, familial colorectal cancer type X; MSI, microsatellite instability; MSS, microsatellite stability; LINE-I, long interspersed element-I; SMD, standardized mean difference; $\mathrm{Cl}$, confidence interval.

\section{Sensitivity analysis and publication bias}

Sensitivity analysis was performed to assess the influence of each individual study. The results suggested that no individual studies significantly affected the pooled SMD indicating a statistically robust result (Figure 4A).

Begg's funnel plot and Egger's linear regression test were performed to assess the publication bias of the included studies. The shape of the funnel plot seemed to be asymmetrical in the dominant model (Figure 4B). Therefore, Egger's test was applied to provide statistical evidence of funnel plot symmetry $(t=-2.77, P=0.022)$. The results suggested that publication bias could not be completely excluded as a factor that influenced the present meta-analysis. Then, trim and fill method was applied. Data from four hypothetical negative unpublished studies were replaced respectively, and the filled funnel plot was symmetrical (Figure 4C). The pooled analysis continued to show a significantly lower level of LINE-1 methylation in cancer patients (SMD: $-3.30 ; 95 \% \mathrm{CI}:-4.50,-2.10 ; P=0.000$ ).

\section{LINE-I hypomethylation is inversely related to the MSI status in CRC}

The mean LINE-1 methylation level was significantly lower in non-microsatellite instability (MSI)-high tumors than in MSIhigh tumors (SMD: $-0.42 ; 95 \% \mathrm{CI}:-0.52,-0.32 ; P=0.000$ ). 


\begin{tabular}{|c|c|c|}
\hline Study ID & SMD $(95 \% \mathrm{Cl})$ & $\%$ weigh \\
\hline \multicolumn{3}{|l|}{ Pyrosequencing } \\
\hline Antelo et $\mathrm{al}^{20}$ (early onset) & $-2.37(-2.81,-1.93)$ & 9.36 \\
\hline Antelo et $\mathrm{al}^{20}$ (Lynch syndrome) & $-3.05(-3.87,-2.23)$ & 8.77 \\
\hline Antelo et $\mathrm{al}^{20}$ (older onset sporadic MSI) & $-1.94(-2.48,-1.39)$ & 9.22 \\
\hline Antelo et $\mathrm{al}^{20}$ (older onset sporadic MSS) & $-1.90(-2.37,-1.43)$ & 9.32 \\
\hline Estecio et $\mathrm{al}^{12}$ & $-11.00(-12.45,-9.55)$ & 7.38 \\
\hline Hur et $\mathrm{al}^{22}$ & $-2.21(-2.61,-1.81)$ & 9.40 \\
\hline Subtotal $\left(I^{2}=96.6 \%, P=0.000\right)$ & $-3.53(-4.76,-2.30)$ & 53.46 \\
\hline \multicolumn{3}{|l|}{ COBRA } \\
\hline Pavicic et al ${ }^{24}$ (sporadic MSS) & $-1.79(-2.23,-1.35)$ & 9.36 \\
\hline Pavicic et al ${ }^{24}$ (sporadic MSI) & $-0.88(-1.29,-0.48)$ & 9.40 \\
\hline Pavicic et al ${ }^{24}$ (Lynch syndrome) & $-1.09(-1.54,-0.63)$ & 9.34 \\
\hline Pavicic et $\mathrm{al}^{24}$ (FCCX) & $-0.57(-1.23,0.10)$ & 9.04 \\
\hline Subtotal $\left(I^{2}=76.3 \%, P=0.005\right)$ & $-1.11(-1.60,-0.62)$ & 37.14 \\
\hline \multicolumn{3}{|l|}{ Multicolor methylight assay } \\
\hline Matsunoki et al ${ }^{21}$ & $0.08(-0.32,0.48)$ & 9.40 \\
\hline Subtotal $\left(I^{2}=. \%, P=.\right)^{*}$ & $0.08(-0.32,0.48)$ & 9.40 \\
\hline Overall $\left(I^{2}=96.6 \%, P=0.000\right)$ & $-2.26(-3.08,-1.45)$ & 100 \\
\hline
\end{tabular}

Figure 3 Forest plot of the SMD of LINE-I methylation levels between colorectal cancer and control groups.

Note: The subgroup analysis was based on methods used to measure global DNA methylation levels. Weights are from random effects analysis. ${ }^{*}$ There is only one study in multicolor methylight assay subgroup, so the $I^{2}$ of the subgroup is blank in the output graph.

Abbreviations: FCCX, familial colorectal cancer type X; MSI, microsatellite instability; MSS, microsatellite stability; LINE-I, long interspersed element-I; SMD, standardized mean difference; $\mathrm{Cl}$, confidence interval.

There was low heterogeneity between studies $\left(I^{2}=48.3 \%\right)$ (Figure 5).

\section{LINE-I methylation levels with clinical and molecular features in CRC}

No difference of LINE-1 methylation levels was found between males and females (SMD: $-0.00 ; 95 \%$ CI: $-0.10,0.10$;
$P=0.974)$. Heterogeneity between studies was low $\left(I^{2}=41.4 \%\right)$ (Figure 6A).

Patients with a family history of CRC had lower LINE-1 methylation level than those without family history (SMD: $-0.23 ; 95 \% \mathrm{CI}:-0.38,-0.08 ; P=0.002$ ). There was no heterogeneity between studies $\left(I^{2}=0.0 \%\right)$ (Figure 6B). 

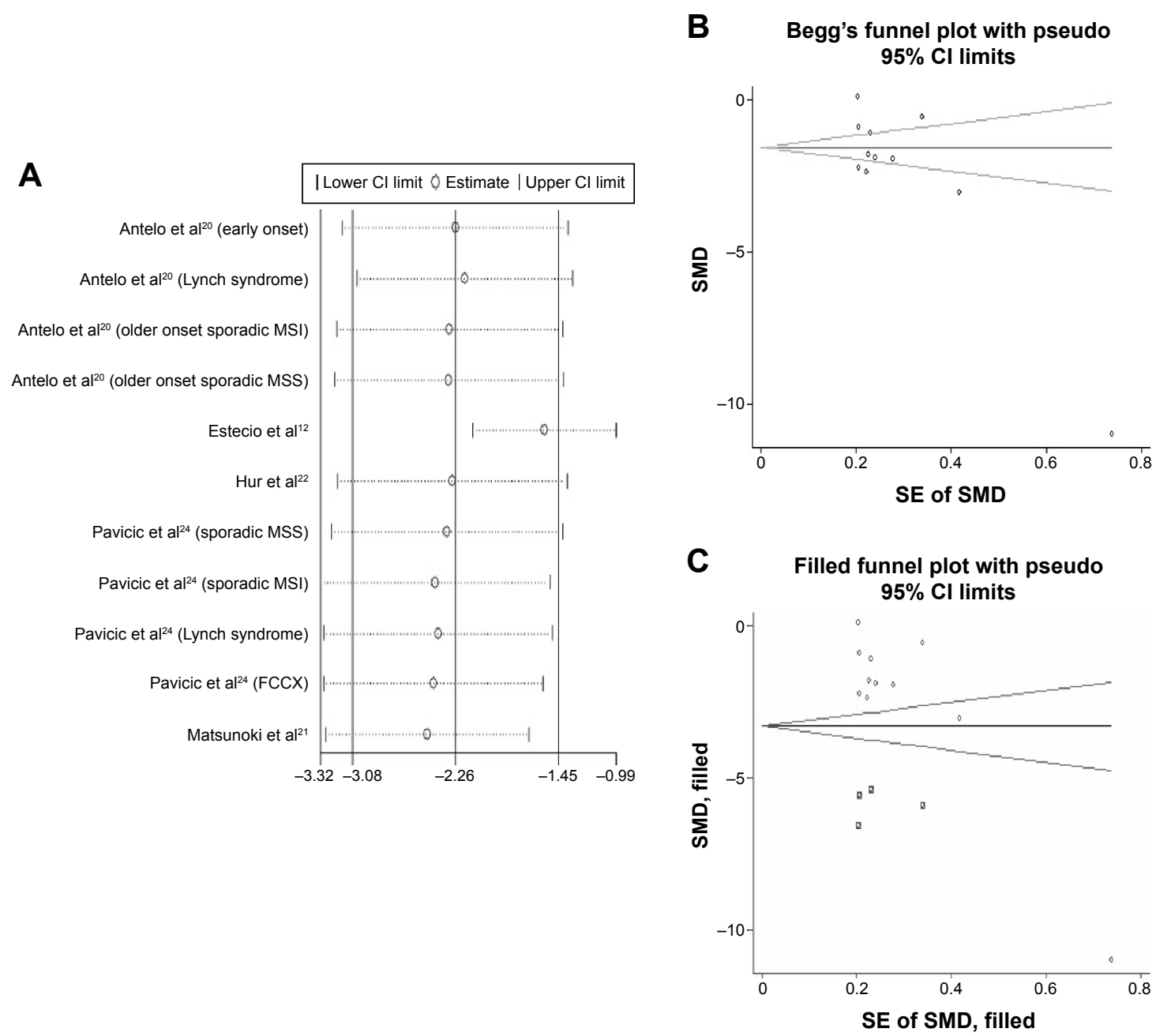

Figure 4 Sensitivity analyses and Begger's funnel plot.

Notes: (A) Sensitivity analyses. Results were computed by omitting each study in turn. Meta-analysis random-effects estimates (exponential form) were used. The two ends of the dotted lines represent the $95 \% \mathrm{Cl}$. (B) Begg's funnel plot of the SMD of LINE-I methylation levels between colorectal cancer and control groups. Each point represents a separate study for the indicated association. Horizontal line indicates the mean magnitude of the effect. (C) Funnel plots with trim and fill. $\bigcirc$ represents identified studies; $\square$ represents estimated missing studies after adjustment for publication bias.

Abbreviations: $\mathrm{CI}$, confidence interval; FCCX, familial colorectal cancer type X; MSI, microsatellite instability; MSS, microsatellite stability; LINE-I, long interspersed element-I; SE, standard error; SMD, standardized mean difference.

There was no difference in LINE-1 methylation levels between patients with a body mass index (BMI) below 30 and those with a BMI above 30 (SMD: $-0.02 ; 95 \%$ CI: -0.19 , $0.16 ; P=0.8)$. There was no heterogeneity between studies $\left(I^{2}=0.0 \%\right)$ (Figure 6C).

The LINE-1 methylation levels in right colon cancer were significantly higher than left colon cancer (SMD: 0.21; 95\% CI: $0.09,0.34 ; P=0.001)$. There was no heterogeneity between studies $\left(I^{2}=0.0 \%\right)$ (Figure $\left.6 \mathrm{D}\right)$.

No difference in LINE-1 methylation levels was found between KRAS-wild-type tumor and KRAS-mutant tumor (SMD: $0.02 ; 95 \% \mathrm{CI}:-0.06,0.11 ; P=0.597)$. There was no heterogeneity between studies $\left(I^{2}=0.0 \%\right)$ (Figure 7A).

No difference in the LINE-1 methylation levels was found between PIK3CA-wild-type tumors and PIK3CA-mutant tumors (SMD: $-0.09 ; 95 \% \mathrm{CI}:-0.24,0.06 ; P=0.247$ ). There was no heterogeneity between studies $\left(I^{2}=0.0 \%\right)$ (Figure 7B).

The LINE-1 methylation levels in tumors that lost the ability to express p21 were significantly lower than p21 expressing tumors (SMD: $-0.34 ; 95 \% \mathrm{CI}:-0.47,-0.21$; $P=0.000)$. There was no heterogeneity between studies $\left(I^{2}=0.0 \%\right)$ (Figure 7C).

\section{Discussion}

Genome-wide DNA hypomethylation is an important epigenetic aberration in human cancer. ${ }^{33}$ As a repetitive DNA retrotransposon, LINE-1 constitutes a large proportion of the human genome. Thus, the level of LINE-1 methylation can approximately represent the level of 


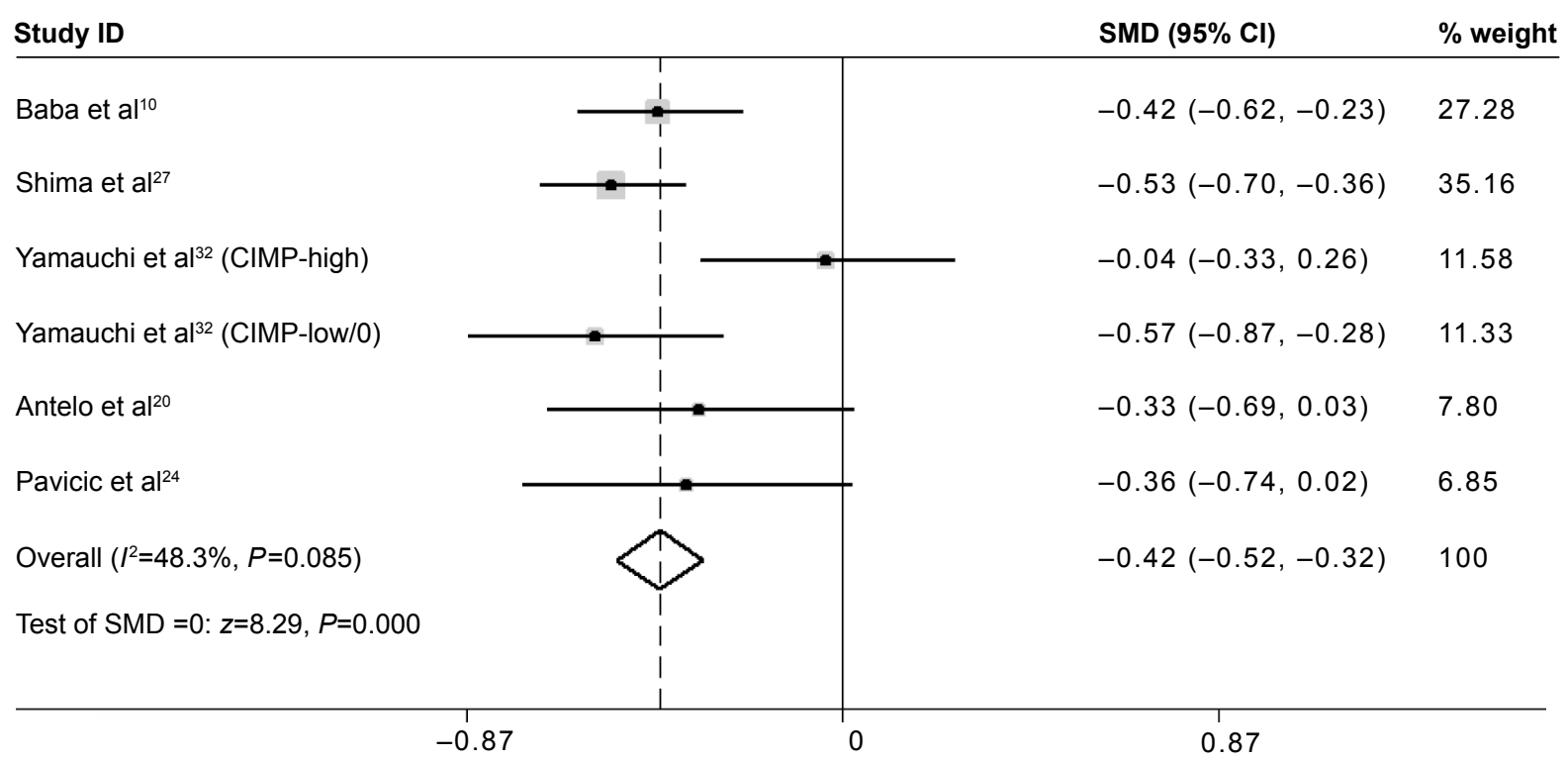

Figure 5 Forest plot of the SMD of LINE-I methylation levels between non-MSI-high tumors and MSI-high tumors.

Abbreviations: Cl, confidence interval; CIMP, CPG island methylator phenotype; LINE-I, long interspersed element-I; MSI, microsatellite instability; SMD, standardized mean difference.

global DNA methylation. ${ }^{34} \mathrm{CRC}$ is an intricate disease resulting from genetic and epigenetic aberrations, including tumor suppressor genes high methylation and global DNA hypomethylation. The methylation level of LINE-1 repeats decline in colon cancer is recognized as a potential prognostic biomarker. However, some of the results remain controversial in several relevant studies. ${ }^{11,13}$ A previous meta-analysis was conducted to evaluate LINE-1 hypomethylation in blood and tissue samples in several types of cancers, but the authors have not fully discussed the association of LINE-1 hypomethylation with clinical and molecular features in CRC, especially those in dispute. ${ }^{14}$ Therefore, we performed the current meta-analysis to investigate more precisely the values of LINE-1 hypomethylation as a biomarker for cancer risk in CRC patients. This meta-analysis concerned 15 unique articles. Two articles included different cancer types, which were separated into substudies. Altogether, there were 21 unique studies with

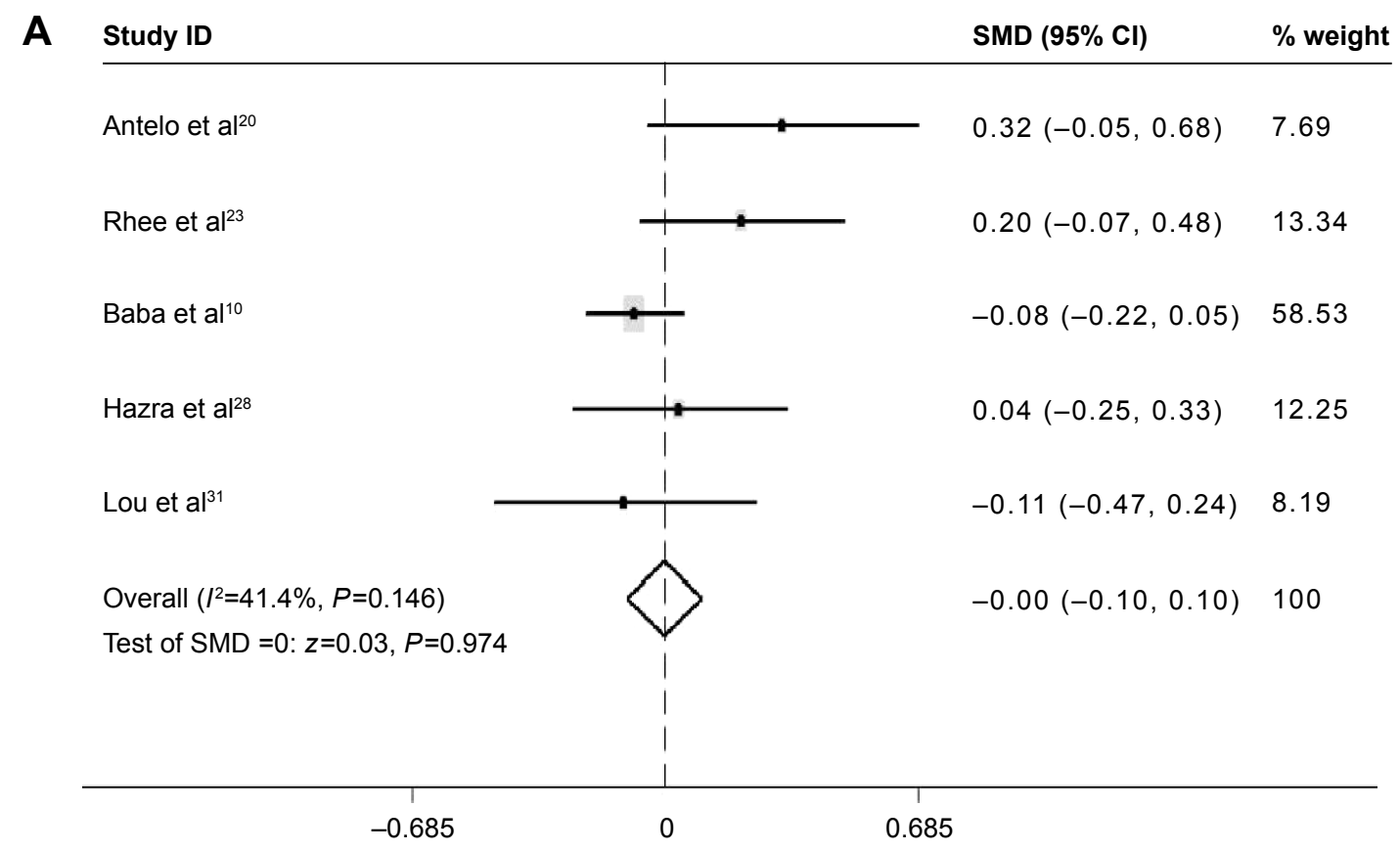

Figure 6 (Continued) 
B Study ID

Antelo et $\mathrm{al}^{20}$

Baba et $\mathrm{al}^{10}$

Overall $\left(I^{2}=0.0 \%, P=0.993\right)$

Test of SMD $=0: z=3.05, P=0.002$
SMD $(95 \% \mathrm{Cl})$

$\%$ weight

7.93

$-0.23(-0.39,-0.08) \quad 92.07$

$-0.23(-0.38,-0.08)$

100

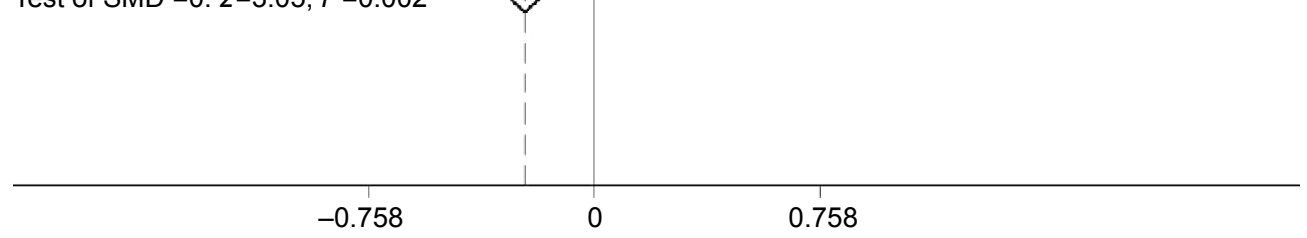

C

Study ID

SMD $(95 \% \mathrm{Cl})$

$\%$ weight

Antelo et $\mathrm{al}^{20}$

(1)

0

\subsection{8}

et al20

Baba et $\mathrm{al}^{10}$

Overall $\left(P^{2}=0.0 \%, P=0.761\right)$

Test of SMD $=0: z=0.20, P=0.838$

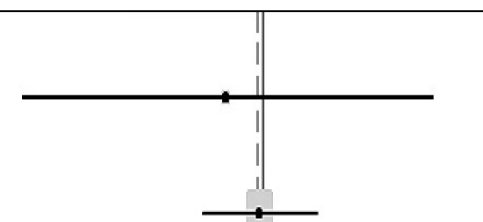

$-0.12(-0.77,0.53)$

7.24

Test of $S M D=0: z=0.20, P=0.838$

$-0.766$

\section{Study ID}

0.766

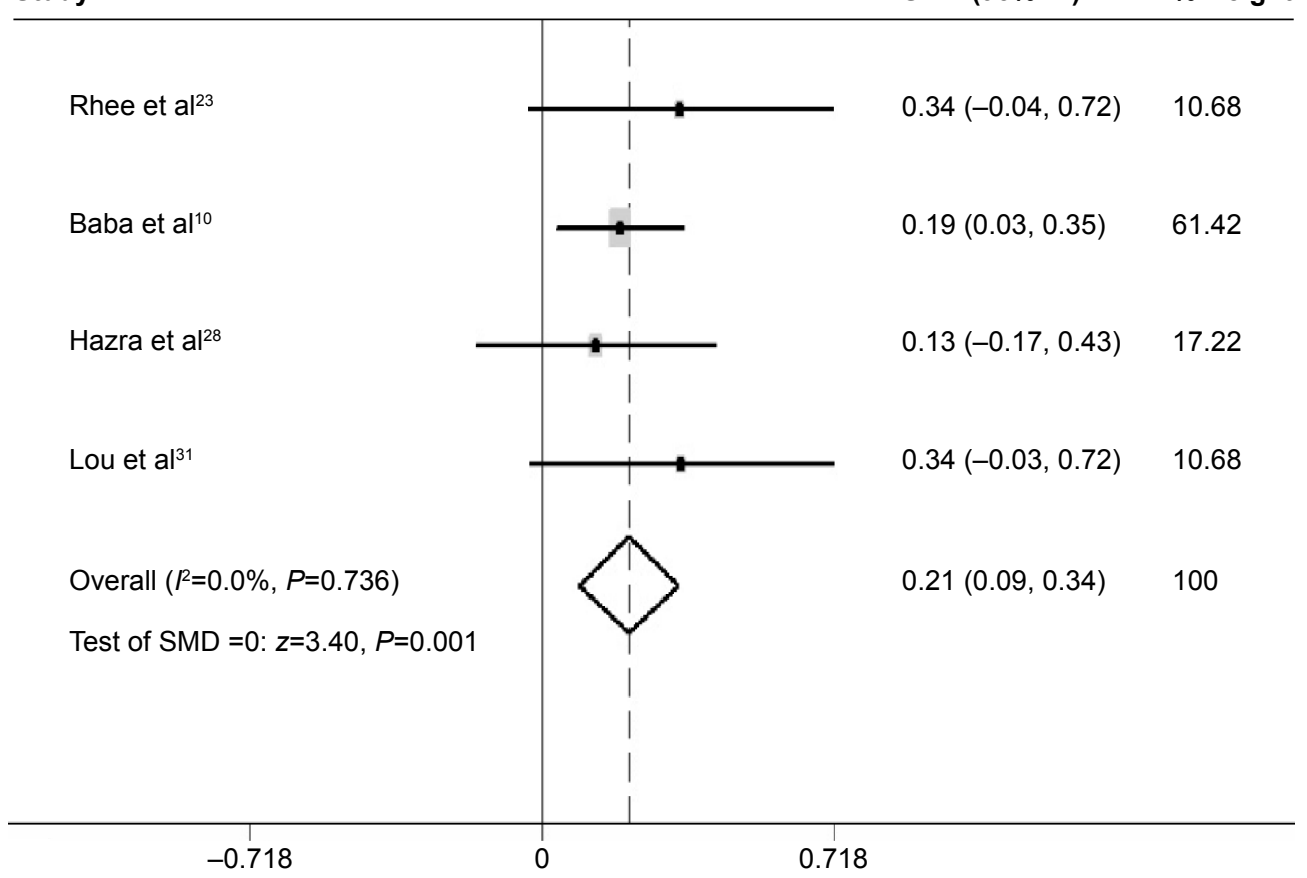

Figure 6 Forest plot of the SMD of LINE-I methylation levels between several clinical features in CRC.

Notes: (A) Forest plot of the SMD of LINE-I methylation levels between males and females. (B) Forest plot of the SMD of LINE-I methylation levels between patients with and without a family history. (C) Forest plot of the SMD of LINE-I methylation levels between patients with BMI values below 30 and above 30 . (D) Forest plot of the SMD of LINE-I methylation levels between patients with right colon cancer and left colon cancer.

Abbreviations: BMI, body mass index; Cl, confidence interval; CRC, colorectal cancer; LINE-I, long interspersed element-I; SMD, standardized mean difference. 
a total of 7396 CRC samples included. According to the results, LINE-1 methylation levels were significantly lower in cancer patients than in control samples (SMD: -2.26 ; 95\% CI: $-3.08,-1.45 ; z=5.44, P=0.000$, Figure 1), which suggested that LINE-1 hypomethylation is a potential biomarker of the risk of CRC. The heterogeneity in the comparisons was significantly high, and this heterogeneity continued in the subgroup analyses conducted by ethnicity. In the subgroup analyses based on the methods used to measure global DNA methylation levels, the heterogeneity between studies in the pyrosequencing subgroups was significantly high $\left(I^{2}=96.6 \%\right)$, and was less, but still high, in the COBRA subgroups $\left(I^{2}=76.3 \%\right)$. Therefore, heterogeneity was a potential problem that may have affected the interpretation of the SMD results.

The MSI pathway is involved in CRC development. ${ }^{35}$ It is caused by the inactivity of the DNA mismatch repair system and associated with the increase of mutation rate in colorectal mucosa cells. ${ }^{36}$ MSI-high CRC presents specific clinical features. Iacopetta et $\mathrm{al}^{37}$ did not found a correlation of methylation levels in LINE-1 with MSI or CpG island methylator phenotype status in CRC. In contrast, Estecio et al ${ }^{12}$ suggested that MSI was the main molecular alteration associated with lack of LINE-1 hypomethylation. Thus, we performed a subgroup analysis to evaluate the relationship between LINE-1 methylation levels and the MSI status. The results showed that the mean LINE-1 methylation level was significantly lower in non-MSIhigh tumors than in MSI-high tumors (SMD: -0.42 ; 95\% CI: $-0.52,-0.32 ; P=0.000)$, which indicated that LINE-1 hypomethylation is inversely related to MSI status. There was low heterogeneity between the studies $\left(I^{2}=48.3 \%\right)$ (Figure 5). Molecular classification of CRC based on MSI status reflects global epigenomic and genomic aberrations in cancer cells, which has great importance during colorectal carcinogenesis. Our results indicated that MSI-high is inversely associated with LINE-1 hypomethylation. Previous studies have proved that global hypomethylation promotes genomic instability which supporting the hypothesis that genome-wide hypomethylation might be involved in the pathway connected with MSI phenomena. ${ }^{38}$ Evidence showed that DNA hypomethylation preceded genomic instability in gastrointestinal cancers, ${ }^{39}$ therefore, LINE-1 hypomethylation is supposed to be a marker of distinction in molecular classification of CRC and might be crucial for cancer prevention and treatment.

Furthermore, subgroup analysis was performed to evaluate the relationship between LINE-1 methylation levels and clinical and molecular features in CRC. Interestingly, the analyses revealed strong associations between LINE-1 hypomethylation and individuals with a family history of CRC (SMD: $-0.23 ; 95 \%$ CI: $-0.38,-0.08 ; P=0.002$ ). The patients with left colon cancer had lower LINE-1 methylation levels than those with right colon cancer (SMD: 0.21; 95\% CI: 0.09, 0.34; $P=0.001)$. There was no heterogeneity between the studies $\left(I^{2}=0.0 \%\right)$. No difference was found between patients with BMI values below 30 and above 30 , or between males and females. These results provide useful information on the independent relations of LINE-1 hypomethylation with various clinical features (family history and tumor location). Researchers have also found patients with LINE-1 hypomethylation presented other specific clinical features, such as early onset, poor prognosis, and lymphocytic reactions. ${ }^{10}$ However, the number of studies is too small to ensure statistical power. Efforts should be made to investigate the relationship between LINE-1 hypomethylation and other clinical manifestations or pathological behavior characteristics. Then, we can further assess whether LINE-1 hypomethylation may produce a distinct subtype of CRCs. LINE-1 hypomethylation was found in tumors that had lost expression of p21 compared with p21 expressing tumors (SMD: -0.34 ; 95\% CI: $-0.47,-0.21$; $P=0.000)$. There was no heterogeneity between the studies $\left(I^{2}=0.0 \%\right)$. p21 plays a key role in regulating the cell cycle. In colon cancer, downregulation of p 21 varied inversely with MSI-high status. ${ }^{40}$ The mechanism of this relation between LINE-1 hypomethylation and p21 remains to be investigated. No difference of LINE-1 methylation levels was found between KRAS-wild-type tumors and KRAS-mutant tumor, or between PIK3CA-wild-type tumors and PIK3CAmutant tumors. The mutation of KRAS and PIK3CA genes has been identified in CRC. Nosho et al showed the loss of O6-methylguanine-DNA methyltransferase (MGMT), but not MGMT promoter methylation, may play a role in the development of PIK3CA mutation. Otherwise, PIK3CA mutation was significantly associated with KRAS mutation and phospho-ribosomal protein S6 expression, and inversely with p53 expression and $\beta$-catenin (CTNNB1) alteration. ${ }^{41}$ It is not clear whether global hypomethylation is related to KRAS or PIK3CA mutation.

However, several limitations of our meta-analysis need to be considered. The number of included studies was small, and the high heterogeneity in the analysis of LINE-1 methylation levels between cancer and normal controls groups was a potential problem in this meta-analysis. In order to resolve this problem, subgroups analysis was performed. The ethnicity and different methods used to measure global DNA methylation levels did not seem to 


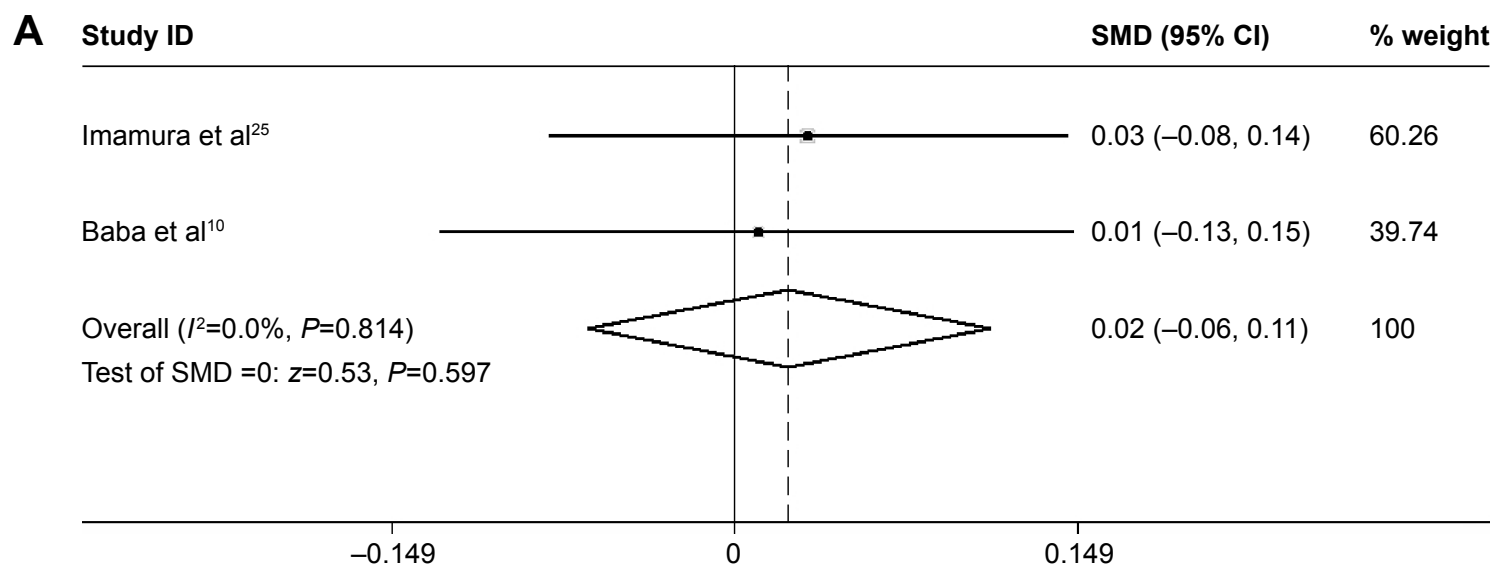

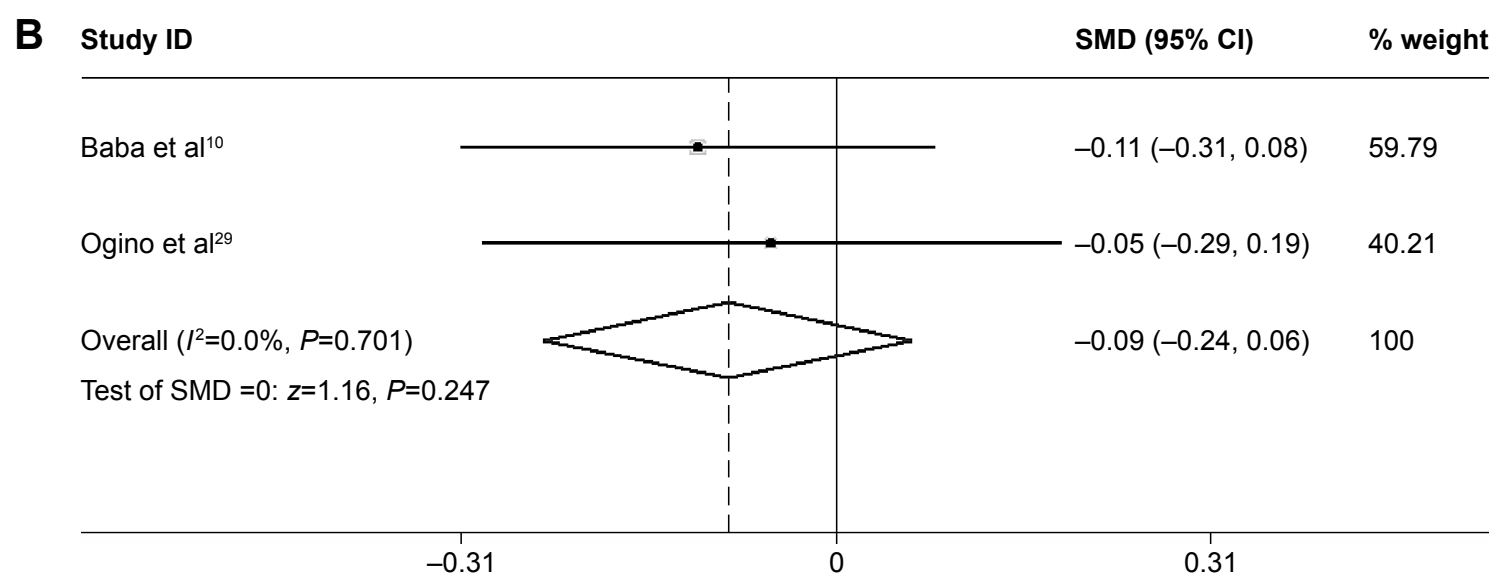

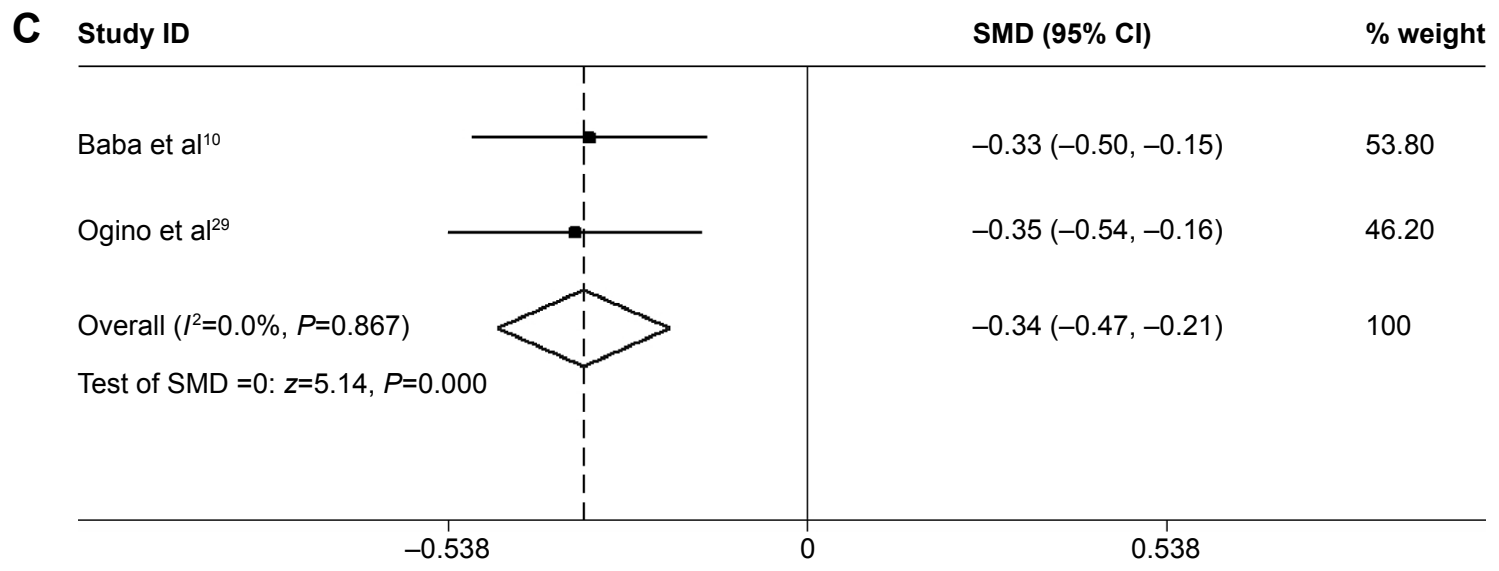

Figure 7 Forest plot of the SMD of LINE-I methylation levels between several molecular features in CRC.

Notes: (A) Forest plot of the SMD of LINE-I methylation levels between KRAS-wild-type tumor and KRAS-mutant tumor. (B) Forest plot of the SMD of LINE-I methylation levels between PIK3CA-wild-type tumor and PIK3CA-mutant tumor. (C) Forest plot of the SMD of LINE-I methylation levels between tumors that lost the ability to express P2I and $\mathrm{p} 2 \mathrm{I}$ expressing tumors.

Abbreviations: $\mathrm{Cl}$, confidence interval; CRC, colorectal cancer; LINE-I, long interspersed element-I; SMD, standardized mean difference.

cause the high heterogeneity. The slight asymmetry funnel plots indicated that the studies included might be influenced by publication bias, and publication bias may not be completely excluded. It might due to the limited numbers of publications recruited. Because of the limitation of the researchers' language ability, the non-English literature could not be reviewed. Besides, some articles enrolled in the analysis might not be with high quality. Trim and 
fill method was used to correct the results. The adjusted estimate was consistent with the previous one. Furthermore, the result of sensitivity analysis showed that there was no single sensitive study in this meta-analysis, which indicated a statistically robust result.

\section{Conclusion}

LINE-1 hypomethylation is associated with various clinical and molecular features of CRC and might be connected with chromosomal instability. Thus, it has the prospect of becoming a biomarker for cancer risk in CRC patients. These results strengthen the importance of LINE-1 hypomethylation in CRC development and open a new venue to explore future cancer prevention strategies. Further investigations in this field would provide deeper insights into the pathogenesis of CRC and clinical implications of LINE-1 methylation.

\section{Acknowledgment}

This work was supported by a grant from the National Natural Science Foundation of China (81372232).

\section{Disclosure}

The authors report no conflicts of interest in this work.

\section{References}

1. Issa JP. CpG island methylator phenotype in cancer. Nat Rev Cancer. 2004;4:988-993.

2. Ushijima T. Detection and interpretation of altered methylation patterns in cancer cells. Nat Rev Cancer. 2005;5:223-231.

3. Esteller M. The necessity of a human epigenome project. Carcinogenesis. 2006;27:1121-1125.

4. Schulz WA. L1 retrotransposons in human cancers. J Biomed Biotechnol. 2006;1:83672.

5. Swergold GD. Identification, characterization, and cell specificity of a human LINE-1 promoter. Mol Cell Biol. 1990;10:6718-6729.

6. Thayer RE, Singer MF, Fanning TG. Undermethylation of specific LINE-1 sequences in human cells producing a LINE-1-encoded protein. Gene. 1993;133:273-277.

7. Hsiung D, Marsit C, Houseman E, et al. Global DNA methylation level in whole blood as a biomarker in head and neck squamous cell carcinoma. Cancer Epidemiol Biomarkers Prev. 2007;16:108-114.

8. Zhu ZZ, Sparrow D, Hou L, et al. Repetitive element hypomethylation in blood leukocyte DNA and cancer incidence, prevalence and mortality in elderly individuals: the Normative Aging Study. Cancer Causes Control. 2011;22:437-447.

9. Wilhelm C, Kelsey K, Butler R, et al. Implications of LINE1 methylation for bladder cancer risk in women. Clin Cancer Res. 2010;16: 1682-1689.

10. Baba Y, Huttenhower C, Nosho K, et al. Epigenomic diversity of colorectal cancer indicated by LINE-1 methylation in a database of 869 tumors. Mol Cancer. 2010;9:125.

11. Ogino S, Kawasaki T, Nosho K, et al. LINE-1 hypomethylation is inversely associated with microsatellite instability and $\mathrm{CpG}$ island methylator phenotype in colorectal cancer. Int J Cancer. 2008; 122:2767-2773.
12. Estecio MR, Gharibyan V, Shen L, et al. LINE-1 hypomethylation in cancer is highly variable and inversely correlated with microsatellite instability. PLoS One. 2007;2:e399.

13. Sunami E, de Maat M, Vu A, et al. LINE-1 hypomethylation during primary colon cancer progression. PLoS One. 2011;6:e18884.

14. Barchitta M, Quattrocchi A, Maugeri A, et al. LINE-1 hypomethylation in blood and tissue samples as an epigenetic marker for cancer risk: a systematic review and meta-analysis. PLoS One. 2014;9:e109478.

15. Higgins J, Thompson SG, Deeks JJ, et al. Measuring inconsistency in meta-analyses. BMJ. 2003;327:557-560.

16. Higgins JP, Thompson SG. Quantifying heterogeneity in a metaanalysis. Stat Med. 2002;21:1539-1558.

17. Begg CB, Mazumdar M. Operating characteristics of a rank correlation test for publication bias. Biometrics. 1994;50:1088-1101.

18. Egger M, Davey Smith G, Schneider M, et al. Bias in meta-analysis detected by a simple, graphical test. BMJ. 1997;315:629-634.

19. Duval S, Tweedie R. Trim and fill: a simple funnel-plot-based method of testing and adjusting for publication bias in meta-analysis. Biometrics. 2000;56:455-463.

20. Antelo M, Balaguer F, Shia J, et al. A high degree of LINE-1 hypomethylation is a unique feature of early-onset colorectal cancer. PLoS One. 2012;7:e45357.

21. Matsunoki A, Kawakami K, Kotake M, et al. LINE-1 methylation shows little intra-patient heterogeneity in primary and synchronous metastatic colorectal cancer. BMC Cancer. 2012;12:574.

22. Hur K, Cejas P, Feliu J, et al. Hypomethylation of long interspersed nuclear element-1 (LINE-1) leads to activation of proto-oncogenes in human colorectal cancer metastasis. Gut. 2014;63:635-646.

23. Rhee YY, Kim MJ, Bae JM, et al. Clinical outcomes of patients with microsatellite-unstable colorectal carcinomas depend on L1 methylation level. Ann Surg Oncol. 2012;19:3441-3448.

24. Pavicic W, Joensuu EI, Nieminen T, et al. LINE-1 hypomethylation in familial and sporadic cancer. $J$ Mol Med (Berl). 2012;90:827-835.

25. Imamura Y, Lochhead P, Yamauchi M, et al. Analyses of clinicopathological, molecular, and prognostic associations of KRAS codon 61 and codon 146 mutations in colorectal cancer: cohort study and literature review. Mol Cancer. 2014;13:135.

26. Morikawa T, Kuchiba A, Liao X, et al. Tumor TP53 expression status, body mass index and prognosis in colorectal cancer. Int $J$ Cancer. 2012;131:1169-1178.

27. Shima K, Morikawa T, Yamauchi M, et al. TGFBR2 and BAX mononucleotidetract mutations, microsatellite instability, and prognosis in 1,072 colorectal cancers. PLoS One. 2011;6:e25062.

28. Hazra A, Fuchs CS, Kawasaki T, et al. Germline polymorphisms in the one-carbon metabolism pathway and DNA methylation in colorectal cancer. Cancer Causes Control. 2010;21:331-345.

29. Ogino S, Nosho K, Shima K, et al. p21 expression in colon cancer and modifying effects of patient age and body mass index on prognosis. Epidemiol Biomarkers Prev. 2009;18:2513-2521.

30. Ogino S, Nosho K, Kirkner GJ, et al. PIK3CA mutation is associated with poor prognosis among patients with curatively resected colon cancer. J Clin Oncol. 2009;27:1477-1484.

31. Lou YT, Chen CW, Fan YC, et al. LINE-1 methylation status correlates significantly to post-therapeutic recurrence in stage III colon cancer patients receiving FOLFOX-4 adjuvant chemotherapy. PLoS One. 2015;28:e123973.

32. Yamauchi M, Morikawa T, Kuchiba A, et al. Assessment of colorectal cancer molecular features along bowel subsites challenges the conception of distinct dichotomy of proximal versus distal colorectum. Gut. 2012;61(6):847-854.

33. Ehrlich M. DNA hypomethylation in cancer cells. Epigenomics. 2009;1:239-259.

34. Cordaux R, Batzer MA. The impact of retrotransposons on human genome evolution. Nat Rev Genet. 2009;10:691-703.

35. Markowitz SD, Bertagnolli MM. Molecular origins of cancer: molecular basis of colorectal cancer. $N$ Engl J Med. 2009;361: 2449-2460. 
36. Thomas DC, Umar A, Kunkel TA. Microsatellite instability and mismatch repair defects in cancer. Mutat Res. 1996;350:201-205.

37. Iacopetta B, Grieu F, Phillips M, et al. Methylation levels of LINE-1 repeats and $\mathrm{CpG}$ island loci are inversely related in normal colonic mucosa. Cancer Sci. 2007;98:1454-1460.

38. Eden A, Gaudet F, Waghmare A, Jaenisch R. Chromosomal instability and tumors promoted by DNA hypomethylation. Science. 2003; 300:455.

39. Suzuki K, Suzuki I, Leodolter A, et al. Global DNA demethylation in gastrointestinal cancer is age dependent and precedes genomic damage. Cancer Cell. 2006;9:199-207.
40. Ogino S, Kawasaki T, Kirkner GJ, et al. Down-regulation of p21 $(\mathrm{CDKN} 1 \mathrm{~A} / \mathrm{CIP} 1)$ is inversely associated with microsatellite instability and $\mathrm{CpG}$ island methylator phenotype (CIMP) in colorectal cancer. J Pathol. 2006;210:147-154.

41. Nosho K, Kawasaki, Ohnishi M, et al. PIK3CA mutation in colorectal cancer: relationship with genetic and epigenetic alterations. Neoplasia. 2008;10:534-541.

\section{Publish your work in this journal}

OncoTargets and Therapy is an international, peer-reviewed, open access journal focusing on the pathological basis of all cancers, potential targets for therapy and treatment protocols employed to improve the management of cancer patients. The journal also focuses on the impact of management programs and new therapeutic agents and protocols on

\section{Dovepress}

\footnotetext{
Submit your manuscript here: http://www.dovepress.com/oncotargets-and-therapy-journal
}

patient perspectives such as quality of life, adherence and satisfaction. The manuscript management system is completely online and includes a very quick and fair peer-review system, which is all easy to use. Visit http://www.dovepress.com/testimonials.php to read real quotes from published authors. 